\title{
Constraints on charmphilic solutions to the muon $g-2$ with leptoquarks
}

\author{
Kamila Kowalska, ${ }^{*}$ Enrico Maria Sessolo, ${ }^{\dagger}$ and Yasuhiro Yamamoto \\ National Centre for Nuclear Research, Hoża 69, 00-681 Warsaw, Poland
}

(Received 23 December 2018; published 11 March 2019)

\begin{abstract}
We derive constraints from flavor and LHC searches on charmphilic contributions to the muon anomalous magnetic moment with a single leptoquark. Only the scalar leptoquarks $S_{1}$ and $R_{2}$ are relevant for the present analysis. We find that for $S_{1}$ some parameter space remains consistent at $2 \sigma$ with the Brookhaven National Laboratory measurement of $(g-2)_{\mu}$, under the assumption that the left-type coupling between the muon and the charm quark is a free parameter. The surviving parameter space is, on the other hand, going to be probed in its entirety at the LHC with $300 \mathrm{fb}^{-1}$ of luminosity or less. All other possibilities are excluded by the LHC dimuon search results in combination with several flavor bounds, which together require one to introduce sizable couplings to the top quark to be evaded.
\end{abstract}

DOI: 10.1103/PhysRevD.99.055007

\section{INTRODUCTION}

The currently running Muon g-2 experiment at Fermilab [1] will measure the anomalous magnetic moment of the muon, $(g-2)_{\mu}$, with precision $0.1 \mathrm{ppm}$. The experiment will be then backed up in the near future by another one at J-PARC [2], designed to reach a comparable sensitivity with a different experimental setup and thus substantially reduce the impact of systematic uncertainties.

These new measurements will play a vital role in constraining the parameter space of the models of new physics invoked in recent years to explain the discrepancy of the previous determination of $(g-2)_{\mu}$ [3], at Brookhaven National Laboratory (BNL), with the Standard Model (SM) expectation. When taking into account recent estimates of the hadronic vacuum polarization uncertainties (see, e.g., $[4,5]$ ), the BNL value was found to be in excess of the SM by approximately 3.5 $: \delta(g-2)_{\mu}=$ $(27.4 \pm 7.6) \times 10^{-10}$, according to the estimate [4], or $\delta(g-2)_{\mu}=(31.3 \pm 7.7) \times 10^{-10}$, according to Ref. [5]

The Large Hadron Collider (LHC) has provided strong constraints on several realistic models that explain the BNL anomaly by introducing new particles not far above the electroweak symmetry-breaking (EWSB) scale. For example, in models based on low-scale supersymmetry [6-8] searches for new physics with 2-3 leptons in the final state

\footnotetext{
*kamila.kowalska@ncbj.gov.pl †enrico.sessolo@ncbj.gov.pl

yasuhiro.yamamoto@ncbj.gov.pl
}

Published by the American Physical Society under the terms of the Creative Commons Attribution 4.0 International license. Further distribution of this work must maintain attribution to the author(s) and the published article's title, journal citation, and DOI. Funded by SCOAP ${ }^{3}$. and large missing energy [9-12] have proven very effective in dramatically restricting the parameter space compatible with $(g-2)_{\mu}$ (see, e.g., Refs. [13-19] for early LHC studies). And, in general, the same constraints apply to virtually any construction where a solution to the $(g-2)_{\mu}$ anomaly involves colorless particles and a dark sector [20]. Even scenarios where $\delta(g-2)_{\mu}$ is obtained without directly involving stable invisible particles, so that one cannot rely on missing energy as a handle to discriminate from the background, are by now strongly constrained $[21,22]$ by the null results of LHC searches with multiple light leptons in the final state $[23,24]$. In general the emerging picture is that one is forced to consider the addition of at least two more states about or above the EWSB scale to explain $\delta(g-2)_{\mu}$ and, at the same time, avoid the tightening LHC bounds.

This is not the case for leptoquarks. As they can boost the value of the anomalous magnetic moment of the muon by coupling with a chirality-flip interaction to a heavy quark, they can yield $\delta(g-2)_{\mu} \sim 10^{-9}$ with acceptable couplings even when their mass is in the TeV range [25-28].

Leptoquarks have attracted significant interest in recent years for several reasons. From the theoretical point of view their existence arises for example as a natural byproduct of the Grand Unification of fundamental interactions. They are also featured in models of supersymmetry without R-parity and in some composite models. But perhaps the main reason that has rendered them a current staple in the high-energy physics literature is the fact that they provide a plausible solution for the recent LHCb Collaboration flavor anomalies (see, e.g., Refs. [29-37] for early explanations based on leptoquarks) if they present non-negligible couplings to the third-generation quarks.

It is possibly for this reason that the most recent studies tackling the $(g-2)_{\mu}$ anomaly with leptoquark solutions 
focus mostly on the couplings to the quarks of the third generation $[35,38,39]$. This choice might also present itself as a matter of convenience, as the third generation is trivially expected to be less constrained phenomenologically than the second. Here, however, we move in the opposite direction and focus instead on the case of charmphilic leptoquarks, i.e., leptoquarks that produce a signal in $(g-2)_{\mu}$ by coupling to the second generation quarks $[27,35]$. Our motivation lies on the fact that, one should not forget, the flavor structure of leptoquark interactions is still largely unconstrained on theoretical grounds, so that their eventual couplings to the SM particles must be inferred (or, in most cases, excluded) by phenomenological analysis. Thus, we apply to leptoquarks the spirit of several recent systematic studies $[20,22,40]$ that, in anticipation of the upcoming data from the Muon g-2 experiment, have confronted renormalizable new physics models consistent with a $(g-2)_{\mu}$ signal with the most recent data from the LHC and complementary input from flavor, precision, and other experiments. In light of the fact that the LHCb anomalies might disappear when further data becomes available, and thus cease to provide a reason for considering predominantly third-generation couplings, we find it perhaps more natural to start with the analysis of the second generation.

We show that almost all realistic and well-motivated charmphilic solutions to the $(g-2)_{\mu}$ anomaly are now excluded by a combination of recent bounds from the LHC [41,42] and a handful of data from flavor experiments, with the exception of narrow slices of the parameter space that will be probed in their entirety with $300 \mathrm{fb}^{-1}$ of luminosity. Thus, if a significant $\delta(g-2)_{\mu}$ deviation from the SM were observed at Fermilab as well, this would mean in this framework that leptoquark couplings to the third generation are required. We provide estimates of the minimal size of third-generation couplings that is necessary to evade all constraints.

The paper is organized as follows. In Sec. II we briefly review the expression for the muon anomalous magnetic moment with one leptoquark at one loop. In Sec. III we introduce the leptoquark models that yield a solution to the BNL $(g-2)_{\mu}$ anomaly with charmphilic couplings, we specify the assumptions involved, and we estimate the required coupling size at $2 \sigma$. In Sec. IV we compute flavor and electroweak (EW) precision constraints on the parameter space of the models. We numerically simulate the relevant LHC searches and present our main results in Sec. V, and we finally conclude in Sec. VI.

\section{THE MUON $g-2$ IN MINIMAL LEPTOQUARK MODELS}

We start by considering the generic Yukawa-type interaction of a scalar leptoquark $S$ with the muon, $\mu$, and one quark or antiquark, $q$ :

$$
\mathcal{L} \supset g_{s} \bar{\mu} q S+g_{p} \bar{\mu} \gamma_{5} q S+\text { H.c. }
$$

It is then well known that $q$ and $S$ contribute at one loop to the calculation of the anomalous magnetic moment of the muon as [28]

$$
\begin{aligned}
\delta(g-2)_{\mu}= & -\frac{N_{c} m_{\mu}^{2}}{8 \pi^{2} m_{S}^{2}}\left\{\frac{m_{q}}{m_{\mu}}\left(\left|g_{s}\right|^{2}-\left|g_{p}\right|^{2}\right)\right. \\
& \times\left[Q_{S} f_{1}(r)+Q_{q} f_{2}(r)\right] \\
& \left.+2\left(\left|g_{s}\right|^{2}+\left|g_{p}\right|^{2}\right)\left[Q_{S} f_{3}(r)+Q_{q} f_{4}(r)\right]\right\},
\end{aligned}
$$

where $m_{\mu}, m_{q}, m_{S}$ are masses of the muon, quark, and leptoquark, respectively; $N_{c}=3$ is a color factor; $Q_{S}, Q_{q}$ are the leptoquark and quark electric charges (with the convention that $Q_{S}+Q_{q}+Q_{\mu}=0$ ); and loop functions of $r=m_{q}^{2} / m_{S}^{2}$ are given by

$$
\begin{gathered}
f_{1}(r)=\frac{1}{2(1-r)^{3}}\left(1-r^{2}+2 r \ln r\right) \\
f_{2}(r)=\frac{1}{2(1-r)^{3}}\left(3-4 r+r^{2}+2 \ln r\right) \\
f_{3}(r)=\frac{1}{12(1-r)^{4}}\left(-1+6 r-3 r^{2}-2 r^{3}+6 r^{2} \ln r\right) \\
f_{4}(r)=\frac{1}{12(1-r)^{4}}\left(2+3 r-6 r^{2}+r^{3}+6 r \ln r\right) .
\end{gathered}
$$

In chiral leptoquark models the relative size of the scalar and pseudoscalar couplings, $g_{s}, g_{p}$, parametrizes the strength of the couplings to the left- and right-chiral component of the muon. We thus redefine $g_{L}=g_{s}+g_{p}$ and $g_{R}=(-1)^{\gamma}\left(g_{s}-g_{p}\right)$, where $\gamma=0$ in cases where $q$ in Eq. (2.1) has the quantum numbers of a SM quark, and $\gamma=1$ if gauge invariance requires $q \rightarrow q^{c}$. Equation (2.2) then becomes, for each generation,

$$
\begin{aligned}
\delta(g-2)_{\mu}= & -\frac{N_{c} m_{\mu}^{2}}{8 \pi^{2} M_{S}^{2}}\left\{\frac{m_{q}}{m_{\mu}}(-1)^{\gamma} \operatorname{Re}\left(g_{L} g_{R}^{*}\right)\right. \\
& \times\left[Q_{S} f_{1}(r)+Q_{q} f_{2}(r)\right] \\
& \left.+\left(\left|g_{L}\right|^{2}+\left|g_{R}\right|^{2}\right)\left[Q_{S} f_{3}(r)+Q_{q} f_{4}(r)\right]\right\},
\end{aligned}
$$

which features explicitly in the first line the chirality-flip term proportional to the quark mass, generated in those models where leptoquarks couple simultaneously to both 
muon chiral states. As was mentioned in Sec. I, by virtue of this coupling the $(g-2)_{\mu}$ anomaly can be resolved in the presence of leptoquarks at the $\mathrm{TeV}$ scale, if the leptoquark is coupled to second- or third-generation quarks, $q=c, t$. This work focuses on the second generation, as we restrict ourselves to charmphilic Yukawa textures that induce a deviation from the SM value parametrized by $\sim m_{c} / m_{\mu} \ln \left(m_{S} / m_{c}\right)$.

Before we proceed to introducing the form of plausible models, let us recall that, in a similar fashion to the scalar case, a vector leptoquark $V^{\rho}$ of mass $m_{V}$ can also contribute to $(g-2)_{\mu}$. Given a generic coupling to one quark and the muon,

$$
\mathcal{L} \supset g_{v} V_{\rho} \bar{q} \gamma^{\rho} \mu+g_{a} V_{\rho} \bar{q} \gamma^{\rho} \gamma_{5} \mu+\text { H.c. }
$$

the one-loop contribution to $(g-2)_{\mu}$ in the limit $m_{q} \ll m_{V}$ reads, for each generation,

$$
\begin{aligned}
\delta(g-2)_{\mu}= & \frac{N_{c} m_{\mu}^{2}}{8 \pi^{2} m_{V}^{2}}\left[\left(\left|g_{v}\right|^{2}+\left|g_{a}\right|^{2}\right)\left(-\frac{4}{3} Q_{q}+\frac{5}{3} Q_{V}\right)\right. \\
& \left.+\left(\left|g_{v}\right|^{2}-\left|g_{a}\right|^{2}\right)\left(Q_{q}-Q_{V}\right) \frac{2 m_{q}}{m_{\mu}}\right],
\end{aligned}
$$

with the convention that $Q_{V}+Q_{q}+Q_{\mu}=0$ [28].

We will briefly come back to the vector leptoquark case in Sec. III. Let us just point out for now that, unlike scalar leptoquark, vector leptoquarks do not induce at one loop a $\sim \ln \left(m_{V} / m_{q}\right)$ enhancement to the $(g-2)_{\mu}$ value, so that they require very large couplings to the secondgeneration quarks if one wishes to accommodate the BNL measurement.

\section{THE MODELS}

Scalar leptoquarks. We begin with scalar leptoquarks. There exist two sole single-leptoquark cases that lead to a mass-enhanced contribution to $(g-2)_{\mu}$ by coupling to both muon chiral states: $\mathrm{SU}(2)_{L}$ singlet $S_{1}$, and $\mathrm{SU}(2)_{L}$ doublet $R_{2}$ [27].

Model 1. Leptoquark $S_{1}$ is characterized by the SM quantum numbers

$$
S_{1}:(\overline{\mathbf{3}}, \mathbf{1}, 1 / 3)
$$

Using the Weyl spinor notation, we introduce $C P$ conserving Yukawa-type couplings to the Lagrangian,

$$
\mathcal{L} \supset Y_{i j}^{D} Q_{i}^{\prime T}\left(-i \sigma_{2}\right) L_{j}^{\prime} S_{1}+Y_{i j}^{S} u_{R i}^{*} e_{R j}^{\prime *} S_{1}+\text { H.c. },
$$

where primed fields are given in the gauge basis, and a sum over the SM generation indices $i, j$ is intended. The quantum numbers of the SM fields and of the leptoquarks considered in this work are summarized in Table I.
TABLE I. Gauge quantum numbers of the SM fields, with generation index $i=1,2,3$, and leptoquarks we consider in this study.

\begin{tabular}{lccc}
\hline \hline Field & $\mathrm{SU}(3)$ & $\mathrm{SU}(2)_{L}$ & $\mathrm{U}(1)_{Y}$ \\
\hline$L_{i}^{\prime}$ & $\mathbf{1}$ & $\mathbf{2}$ & $-1 / 2$ \\
$e_{R i}^{\prime}$ & $\mathbf{1}$ & $\mathbf{1}$ & 1 \\
$Q_{i}^{\prime}$ & $\mathbf{3}$ & $\mathbf{2}$ & $1 / 6$ \\
$u_{R i}^{\prime}$ & $\overline{\mathbf{3}}$ & $\mathbf{1}$ & $-2 / 3$ \\
$d_{R i}^{\prime}$ & $\overline{\mathbf{3}}$ & $\mathbf{1}$ & $1 / 3$ \\
$H$ & $\mathbf{1}$ & $\mathbf{2}$ & $1 / 2$ \\
$S_{1}$ & $\overline{\mathbf{3}}$ & $\mathbf{1}$ & $1 / 3$ \\
$R_{2}$ & $\mathbf{3}$ & $\mathbf{2}$ & $7 / 6$ \\
$U_{1}^{\rho}$ & $\mathbf{3}$ & $\mathbf{1}$ & $2 / 3$ \\
\hline \hline
\end{tabular}

Note that the quantum number can allow one to write down Lagrangian terms of the form $\mathcal{L} \supset \lambda_{i j}^{D} Q_{i}^{\prime} Q_{j}^{\prime} S_{1}^{*}+$ $\lambda_{i j}^{S} u_{R i}^{\prime} d_{R j}^{\prime} S_{1}+$ H.c., which can lead to fast proton decay. In order to forbid these dangerous terms, we assume the existence of a symmetry (for example, conservation of baryon and/or lepton number).

After EWSB one can rotate the Lagrangian to the quark mass basis and write down the couplings to the secondgeneration leptons, required for $(g-2)_{\mu}$ :

$\mathcal{L} \supset\left(-\tilde{Y}_{i}^{L} u_{L i} \mu_{L}+\hat{Y}_{i}^{L} d_{L i} \nu_{\mu}+Y_{i}^{R} u_{R i}^{*} \mu_{R}^{*}\right) S_{1}+$ H.c.,

where nonprimed fields indicate mass eigenstates, we have defined $Q_{i}^{T} \equiv\left(u_{L i}, d_{L i}\right), L_{i} \equiv\left(\nu_{i}, e_{L i}\right)^{T}$, and the $L$-type couplings are related to each other via the CabibboKobayashi-Maskawa (CKM) matrix, $\tilde{Y}_{i}^{L}=\hat{Y}_{k}^{L}\left(V_{\mathrm{CKM}}^{\dagger}\right)_{k i}$.

We now implement the charmphilic assumption for the $Y_{i}^{R}$ Yukawa couplings by imposing $Y_{1}^{R}=Y_{3}^{R}=0$. The contribution to $(g-2)_{\mu}$ is read off from Eq. (2.7), where $\gamma=1, g_{L}=-\tilde{Y}_{2}^{L}, g_{R}=Y_{2}^{R}, m_{q}=m_{c}, Q_{q}=2 / 3$, and $Q_{S}=1 / 3$. It leads to the $2 \sigma$ bound (we use the estimate in Ref. [4])

$$
\begin{aligned}
3.1 \times 10^{-2}\left(\frac{m_{S_{1}}}{\mathrm{TeV}}\right)^{2} & \leq \operatorname{Re}\left(\tilde{Y}_{2}^{L} Y_{2}^{R *}\right)\left(1+0.17 \ln \frac{m_{S_{1}}}{\mathrm{TeV}}\right) \\
& \leq 11 \times 10^{-2}\left(\frac{m_{S_{1}}}{\mathrm{TeV}}\right)^{2} .
\end{aligned}
$$

For the $L$-type couplings, which are related by gauge invariance through the CKM matrix, we base our phenomenological analysis on two limiting cases: the up origin, featuring $\tilde{Y}_{1}^{L}=\tilde{Y}_{3}^{L}=0$; and the down origin, featuring $\hat{Y}_{1}^{L}=\hat{Y}_{3}^{L}=0$.

In the up-origin case the couplings $\hat{Y}_{1}^{L}, \hat{Y}_{2}^{L}$, and $\hat{Y}_{3}^{L}$ to the down, strange, and bottom quark, respectively, will be generated by multiplication with the CKM matrix. As a consequence, the $L$-type coupling $\tilde{Y}_{2}^{L}$ becomes subject to flavor bounds from, e.g., the $K^{+} \rightarrow \pi^{+} \nu \bar{\nu}$ rare decay. 
On the other hand, in the down-origin case, the couplings $\tilde{Y}_{1}^{L}, \tilde{Y}_{2}^{L}$, and $\tilde{Y}_{3}^{L}$ to the up, charm, and top quark, respectively, are CKM-generated and thus the $L$-type coupling $\hat{Y}_{2}^{L}$ can be bounded by processes like $D^{0} \rightarrow \mu^{+} \mu^{-}$. Flavorchanging neutral current (FCNC) constraints do not apply, however, to the charmphilic $R$-type coupling $Y_{2}^{R}$. If it is required to be large in order to satisfy Eq. (3.4), it can be probed directly by collider searches at the LHC.

Model 2. Leptoquark $R_{2}$ is characterized by the SM quantum numbers

$$
R_{2}:(3,2,7 / 6)
$$

The gauge-invariant Yukawa interactions read in this case

$$
\mathcal{L} \supset Y_{i j}^{L} L_{i}^{\prime T}\left(-i \sigma_{2}\right) R_{2} u_{R j}^{\prime}+Y_{i j}^{R} Q_{i}^{\prime \dagger} R_{2} e_{R j}^{\prime *}+\text { H.c. },
$$

where a sum over family indices is intended.

In the quark mass basis the couplings to the secondgeneration leptons are then given by

$$
\begin{aligned}
\mathcal{L} \supset & Y_{i}^{L} u_{R i}\left[\mu_{L} s_{(5 / 3)}-\nu_{\mu} s_{(2 / 3)}\right]+\tilde{Y}_{i}^{R} u_{L i}^{*} \mu_{R}^{*} s_{(5 / 3)} \\
& +\hat{Y}_{i}^{R} d_{L i}^{*} \mu_{R}^{*} s_{(2 / 3)}+\text { H.c. },
\end{aligned}
$$

where the scalar fields $s_{(5 / 3)}$ and $s_{(2 / 3)}$ belong to the $R_{2}$ doublet (electric charge in parentheses), and again we work under the charmphilic assumption, $Y_{1}^{L}=Y_{3}^{L}=0$.

The contribution to $(g-2)_{\mu}$ in Model 2 is given in Eq. (2.7), where $\gamma=0, g_{L}=Y_{2}^{L}, g_{R}=\tilde{Y}_{2}^{R}, m_{q}=m_{c}$, $Q_{q}=-2 / 3$, and $Q_{S}=5 / 3$. It leads to the $2 \sigma$ bound

$$
\begin{aligned}
2.7 \times 10^{-2}\left(\frac{m_{R_{2}}}{\mathrm{TeV}}\right)^{2} & \leq-\operatorname{Re}\left(\tilde{Y}_{2}^{R} Y_{2}^{L *}\right)\left(1+0.15 \ln \frac{m_{R_{2}}}{\mathrm{TeV}}\right) \\
& \leq 9.5 \times 10^{-2}\left(\frac{m_{R_{2}}}{\mathrm{TeV}}\right)^{2} .
\end{aligned}
$$

We introduce for the $R$-type couplings an up-origin and a down-origin scenario, in analogy to Model 1. In the uporigin case, we generate couplings to the down and bottom quarks, which render the $R$-type coupling $\tilde{Y}_{2}^{R}$ subject to constraints, e.g., from the decay $K_{L} \rightarrow \mu^{+} \mu^{-}$. In the downorigin case, generated $R$-type couplings to the up-type quarks can be constrained by the measurement of the $D^{0} \rightarrow \mu^{+} \mu^{-}$transition.

Vector leptoquarks. We finally tackle the case of a vector leptoquark at the $\mathrm{TeV}$ scale as the primary responsible for the $(g-2)_{\mu}$ anomaly. In general, an appropriate treatment of vector states cannot be carried out without some starting assumption on the nature of the UV completion that gives rise to the leptoquark itself. In fact, the UV completion might produce additional states lighter than the vector leptoquark (as happens, e.g., in "composite" models $[43,44])$, which, when accounted for, can reduce the relevance of the heavier vector for the computation of the observable in question. If, on the other hand, the vector leptoquark is a gauge boson, the appropriate treatment of gauge anomalies should be factored in.

In recent years, several studies [45-49] on UV completions based on Pati-Salam constructions have pointed out that an $\mathrm{SU}(2)_{L}$ singlet vector leptoquark $U_{1}^{\rho}$ can emerge as the lightest state of the new physics spectrum around the $\mathrm{TeV}$ scale. Because of its gauge quantum numbers, however, a leptoquark $U_{1}^{\rho}:(\mathbf{3}, \mathbf{1}, 2 / 3)$ coupled to secondgeneration quarks does not produce a mass-enhanced contribution to $(g-2)_{\mu}$ with the charm quark in the loop, but rather with the strange quark.

After rotating to the mass basis the Lagrangian, in fact, reads

$$
\begin{aligned}
\mathcal{L} \supset \tilde{g}_{i}^{L} u_{L i}^{*} U_{1 \rho} \bar{\sigma}^{\rho} \nu_{\mu}+\hat{g}_{i}^{L} d_{L i}^{*} U_{1 \rho} \bar{\sigma}^{\rho} \mu_{L} \\
\quad+g_{i}^{R} d_{R i} U_{1 \rho} \sigma^{\rho} \mu_{R}^{*}+\text { H.c. },
\end{aligned}
$$

in terms of generic gauge couplings $\tilde{g}_{i}^{L}, \hat{g}_{i}^{L}, g_{i}^{R}$. The contribution to $(g-2)_{\mu}$ is obtained from the sum over 3 generations in Eq. (2.9) after the following definitions: $g_{v 1,3}=-g_{a 1,3}=\hat{g}_{1,3}^{L} / 2, g_{v 2}=\left(g_{2}^{R}+\hat{g}_{2}^{L}\right) / 2, g_{a 2}=\left(g_{2}^{R}-\hat{g}_{2}^{L}\right) / 2$, $m_{q}=m_{d, s, b}, Q_{q}=1 / 3$, and $Q_{V}=2 / 3$.

Since the contribution to $(g-2)_{\mu}$ is not subject to a $\ln \left(m_{V} / m_{q}\right)$ enhancement and, additionally, $m_{s} / m_{\mu} \approx 0.9$, upholding the $2 \sigma$ bound from $\delta(g-2)_{\mu}$ requires either small mass or significant couplings:

$$
\left|g_{i}^{L}\right|=\left|g_{i}^{R}\right| \gtrsim 2.0\left(\frac{m_{U_{1}^{\rho}}}{\mathrm{TeV}}\right),
$$

where we have indicated all $L$-type couplings generically with $g_{i}^{L}$.

The current pair-production LHC mass bounds [50], recast for the case of the $U_{1}^{\rho}$ leptoquark [51], yield $m_{U_{1}^{\rho}}>1.5 \mathrm{TeV}$. Relation (3.10) thus implies that, in order to explain the $(g-2)_{\mu}$ anomaly, strangephilic vector leptoquarks must have coupling size of about 3 or greater. Recent LHC dimuon analyses, to which we will come back in Sec. V, when applied specifically to this case have shown [51,52] that a coupling to the strange quark of size 3 or more is excluded. Therefore, we will not consider the vector case any further in this work.

\section{FLAVOR AND ELECTROWEAK PRECISION CONSTRAINTS}

Due to their a priori unconstrained flavor structure, leptoquarks can generate sizable contributions in some flavor observables, which in turn can lead to undesirable flavor signals. After integrating out the leptoquarks, the models introduced in Sec. III produce the tree-level effective Lagrangians 


$$
\begin{aligned}
\mathcal{L}_{S 1}^{\text {eff }}= & \frac{1}{2 m_{S 1}^{2}}\left\{\tilde{Y}_{i j}^{L} \tilde{Y}_{k l}^{L *}\left(\bar{u}_{L i} \gamma^{\mu} u_{L k}\right)\left(\bar{e}_{L j} \gamma_{\mu} e_{L l}\right)+\hat{Y}_{i j}^{L} \hat{Y}_{k l}^{L *}\left(\bar{d}_{L i} \gamma^{\mu} d_{L k}\right)\left(\bar{\nu}_{L j} \gamma_{\mu} \nu_{L l}\right)\right. \\
& +Y_{i j}^{R} Y_{k l}^{R *}\left(\bar{u}_{R i} \gamma^{\mu} u_{R k}\right)\left(\bar{e}_{R j} \gamma_{\mu} e_{R l}\right)+\tilde{Y}_{i j}^{L} \hat{Y}_{k l}^{L *}\left(\bar{u}_{L i} \gamma^{\mu} d_{L k}\right)\left(\bar{e}_{L j} \gamma_{\mu} \nu_{L l}\right) \\
& +\frac{\tilde{Y}_{i j}^{L} Y_{k l}^{R *}}{4}\left[-4\left(\bar{u}_{L i} u_{R k}\right)\left(\bar{e}_{L j} e_{R l}\right)+\left(\bar{u}_{L i} \sigma^{\mu \nu} u_{R k}\right)\left(\bar{e}_{L j} \sigma_{\mu \nu} e_{R l}\right)\right] \\
& \left.+\frac{\hat{Y}_{i j}^{L} Y_{k l}^{R *}}{4}\left[-4\left(\bar{d}_{L i} u_{R k}\right)\left(\bar{\nu}_{L j} e_{R l}\right)+\left(\bar{d}_{L i} \sigma^{\mu \nu} u_{R k}\right)\left(\bar{\nu}_{L j} \sigma_{\mu \nu} e_{R l}\right)\right]+\cdots\right\}, \\
\mathcal{L}_{R 2}^{\text {eff }}=- & \frac{1}{2 m_{s_{(5 / 3)}}^{2}}\left\{\tilde{Y}_{i j}^{R} \tilde{Y}_{k l}^{R *}\left(\bar{u}_{L i} \gamma^{\mu} u_{L k}\right)\left(\bar{e}_{R l} \gamma_{\mu} e_{R j}\right)+Y_{i j}^{L} Y_{k l}^{L *}\left(\bar{u}_{R i} \gamma^{\mu} u_{R k}\right)\left(\bar{e}_{L l} \gamma_{\mu} e_{L j}\right)\right. \\
+ & \left.\frac{Y_{i j}^{L} \tilde{Y}_{k l}^{R *}}{4}\left[4\left(\bar{u}_{R i} u_{L k}\right)\left(\bar{e}_{R l} e_{L j}\right)+\left(\bar{u}_{R i} \sigma_{\mu \nu} u_{L k}\right)\left(\bar{e}_{R l} \sigma^{\mu \nu} e_{L j}\right)\right]+\cdots\right\} \\
- & \frac{1}{2 m_{s_{(2 / 3)}}^{2}}\left\{\hat{Y}_{i j}^{R} \hat{Y}_{k l}^{R *}\left(\bar{d}_{L i} \gamma^{\mu} d_{L k}\right)\left(\bar{e}_{R l} \gamma_{\mu} e_{R j}\right)+Y_{i j}^{L} Y_{k l}^{L *}\left(\bar{u}_{R i} \gamma^{\mu} u_{R k}\right)\left(\bar{\nu}_{L l} \gamma_{\mu} \nu_{L j}\right)\right. \\
& \left.-\frac{Y_{i j}^{L} \hat{Y}_{k l}^{R *}}{4}\left[4\left(\bar{u}_{R i} d_{L k}\right)\left(\bar{e}_{R l} \nu_{L j}\right)+\left(\bar{u}_{R i} \sigma_{\mu \nu} d_{L k}\right)\left(\bar{e}_{R l} \sigma^{\mu \nu} \nu_{L j}\right)\right]+\cdots\right\}
\end{aligned}
$$

where the ellipsis indicate the Hermitian conjugate of the shown operators if they are not self-conjugate, and, with a slight abuse of notation, we generalize the couplings of Sec. III to matrices such that $\tilde{Y}_{i}^{L} \equiv \tilde{Y}_{i 2}^{L}$ and so on. Since the $\tilde{Y}_{i j}^{L, R}$ and $\hat{Y}_{i j}^{L, R}$ Yukawa couplings are related to each other via the CKM matrix, some FCNCs are inevitably induced at the tree level.

Leptoquark $R_{2}$ belongs to a doublet representation and consists of two colored scalars, $s_{(5 / 3)}$ and $s_{(2 / 3)}$. Their Yukawa couplings are related to each other, but in principle experimental constraints on the couplings depend on the scalars' individual masses. The mass difference of the doublet states violates the custodial symmetry, so that the $T$-parameter is sensitive to it. The leptoquark contribution to the $T$-parameter reads

$$
\begin{aligned}
\Delta T= & \frac{3}{16 \pi m_{Z}^{2} s_{W}^{2} c_{W}^{2}} \\
& \times\left(m_{(5 / 3)}^{2}+m_{(2 / 3)}^{2}-\frac{2 m_{(5 / 3)}^{2} m_{(2 / 3)}^{2}}{m_{(5 / 3)}^{2}-m_{(2 / 3)}^{2}} \ln \frac{m_{(5 / 3)}^{2}}{m_{(2 / 3)}^{2}}\right) \\
= & \frac{\Delta m^{2}}{4 \pi m_{Z}^{2} s_{W}^{2} c_{W}^{2}}\left[1+O\left(\frac{\Delta m^{2}}{m_{(2 / 3)}^{2}}\right)\right]
\end{aligned}
$$

where $m_{Z}$ is the $Z$ boson mass, $s_{W}, c_{W}$ are the sine and cosine of the Weinberg angle, and $\Delta m=m_{(5 / 3)}-m_{(2 / 3)}$, see Ref. [53].
Given the observed limit, $\Delta T=0.09 \pm 0.13$ [54], at $1 \sigma$ the mass difference must be bounded by

$$
|\Delta m|=2 s_{W} c_{W} \sqrt{\pi|\Delta T|} m_{Z} \lesssim 63 \mathrm{GeV} .
$$

Since the typical mass region studied in this paper is a few $\mathrm{TeV}$, we can easily neglect this difference, given the bound on $\Delta T$. Thus, we perform the analysis in this paper in the approximation where the two states are degenerate.

\section{A. Down origin}

The down-origin ansatz makes a solution for the $(g-2)_{\mu}$ anomaly via charmphilic leptoquark inconsistent with the measurement of the branching ratio of the rare flavor process $D^{0} \rightarrow \mu^{+} \mu^{-}[35,55]$.

The most recent measurement [56] of the branching ratio at $\mathrm{LHCb}$ reads, at the $95 \%$ C.L.,

$$
\operatorname{Br}\left(D^{0} \rightarrow \mu^{+} \mu^{-}\right)<7.6 \times 10^{-9} .
$$

We write the branching ratio in the most general form as $[57,58]$

$$
\begin{aligned}
\operatorname{Br}\left(D^{0} \rightarrow \mu^{+} \mu^{-}\right)= & \tau_{D} \frac{f_{D}^{2} m_{D}^{3}}{256 \pi} \frac{m_{D}^{2}}{m_{c}^{2}}\left[\left|C_{S R R}^{D}-C_{S L L}^{D}\right|^{2}\right. \\
& +\mid C_{S R R}^{D}+C_{S L L}^{D}-\frac{2 m_{\mu} m_{c}}{m_{D}^{2}} \\
& \left.\times\left.\left(C_{V L L}^{D}+C_{V R R}^{D}-C_{V R L}^{D}-C_{V L R}^{D}\right)\right|^{2}\right],
\end{aligned}
$$


TABLE II. Numerical parameters relative to the calculation of $\operatorname{Br}\left(D^{0} \rightarrow \mu^{+} \mu^{-}\right)$, Eq. (4.7). We take the muon mass and $\hbar$ as in Ref. [60], whereas other constants are set at their Particle Data Group (PDG) value [61].

\begin{tabular}{lc}
\hline \hline$\hbar$ & $6.58 \times 10^{-25} \mathrm{GeV} \mathrm{sec}$ \\
$m_{\mu}$ & $106 \mathrm{MeV}$ \\
$\lambda$ & 0.226 \\
\hline \hline$\tau_{D}$ & $4.10 \times 10^{-13} \mathrm{sec}$. \\
$m_{D}$ & $1.86 \mathrm{GeV}$ \\
$f_{D}$ & $212 \mathrm{MeV}$ \\
$m_{c}$ & $1.28 \mathrm{GeV}$ \\
\hline \hline
\end{tabular}

where the parameters relative to $D$ meson properties are featured in Table II. The SM contribution is dominated by the long-distance effect which is smaller than $10^{-12}$ [59].

As a function of the couplings of Model 1, the Wilson coefficients can be expressed as

$$
\begin{aligned}
C_{V L L}^{D}=-\frac{1}{2 m_{S 1}^{2}} \tilde{Y}_{1}^{L} \tilde{Y}_{2}^{L *} & =-\frac{1}{2 m_{S 1}^{2}} \frac{\lambda}{1-\lambda^{2} / 2}\left|\tilde{Y}_{2}^{L}\right|^{2}, \\
C_{V R R}^{D} & =-\frac{1}{2 m_{S 1}^{2}} Y_{1}^{R} Y_{2}^{R *}, \\
C_{S L L}^{D}=\frac{\eta_{\mathrm{QCD}}}{2 m_{S 1}^{2}} \tilde{Y}_{1}^{L} Y_{2}^{R *} & =\frac{\eta_{\mathrm{QCD}}}{2 m_{S 1}^{2}} \frac{\lambda}{1-\lambda^{2} / 2} \tilde{Y}_{2}^{L} Y_{2}^{R *}, \\
C_{S R R}^{D} & =\frac{\eta_{\mathrm{QCD}}}{2 m_{S 1}^{2}} Y_{1}^{R} \tilde{Y}_{2}^{L *},
\end{aligned}
$$

where $\eta_{\mathrm{QCD}}$ stands for the QCD running effect of the scalar operators, $C_{S X X}\left(m_{c}\right) / C_{S X X}\left(m_{S_{1}}\right)$, and we used the fact that, in terms of the Wolfenstein parameters, $\tilde{Y}_{1}^{L}=\lambda \hat{Y}_{2}^{L}$ and $\tilde{Y}_{2}^{L}=\left(1-\lambda^{2} / 2\right) \hat{Y}_{2}^{L}$. Since $\eta_{\mathrm{QCD}}=1.99$ for $m_{S_{1}}=$ $1.5 \mathrm{TeV}$ [58], we assume $\eta_{\mathrm{QCD}} \approx 2$ in the following analysis.

Recall from Sec. III that, under the charmphilic ansatz, Yukawa coupling $Y_{1}^{R}$ is identically set to zero, so that Eq. (4.6) can be used to derive an upper bound on the product $\tilde{Y}_{2}^{L} Y_{2}^{R *}$. At this point, however, one could wonder whether the same bound could be relaxed for specific nonzero values of $Y_{1}^{R}$, due to a cancellation between different Wilson coefficients. To derive the bound, it is thus worth treating the coupling $Y_{1}^{R}$, which does not contribute to $(g-2)_{\mu}$, as a free parameter.

To analyze the impact of $Y_{1}^{R}$, we calculate the minimum of the branching ratio with respect to $Y_{1}^{R}$ and obtain

$$
\begin{aligned}
\operatorname{Br}\left(D^{0} \rightarrow \mu^{+} \mu^{-}\right)_{\min }= & \frac{5.4 \times 10^{-2}}{\pi} \tau_{D} m_{D} \\
& \times\left(\frac{f_{D} m_{D}^{2}}{32 m_{c} m_{S 1}^{2}}\left|\tilde{Y}_{2}^{L} Y_{2}^{R *}\right|\right)^{2},
\end{aligned}
$$

under the assumption that $\left|\tilde{Y}_{2}^{L} / Y_{2}^{R}\right| \ll 1$. The above equation thus yields a conservative bound on the product of the charmphilic couplings, which reads

$$
\left|\tilde{Y}_{2}^{L} Y_{2}^{R *}\right| \leq 1.1 \times 10^{-2}\left(\frac{m_{S_{1}}}{\mathrm{TeV}}\right)^{2} .
$$

Note that, since the rare $D$ decay bound constrains the absolute value of the Yukawa coupling product, it cannot be avoided by introducing the imaginary parts of the couplings. By comparing Eqs. (4.13) and (3.4), we see that $D^{0}$ decay excludes the entire $2 \sigma$ region of $\delta(g-2)_{\mu}$ for the downorigin scenario of Model 1. A calculation along similar lines, with $\tilde{Y}_{i}^{L} \leftrightarrow \tilde{Y}_{i}^{R}, \hat{Y}_{i}^{L} \leftrightarrow \hat{Y}_{i}^{R}, Y_{i}^{R} \leftrightarrow Y_{i}^{L}$ in Eq. (4.7), leads to the exclusion of the down-origin case in Model 2.

One could further wonder, at this point, about how large an eventual coupling to the third generation should be in order to avoid full exclusion of the charmphilic scenario. We can evaluate this for Model 1 by recalling that, in the presence of the third generation, Eq. (3.4) is modified into

$$
\begin{aligned}
3.1 \times 10^{-2}\left(\frac{m_{S_{1}}}{\mathrm{TeV}}\right)^{2} & \leq a_{c} \operatorname{Re}\left(\tilde{Y}_{2}^{L} Y_{2}^{R *}\right)+20.7 a_{t} \operatorname{Re}\left(\tilde{Y}_{3}^{L} Y_{3}^{R *}\right) \\
& \leq 11 \times 10^{-2}\left(\frac{m_{S_{1}}}{\mathrm{TeV}}\right)^{2}
\end{aligned}
$$

where $\quad a_{c}=1+0.17 \ln \left(m_{S_{1}} / \mathrm{TeV}\right) \quad$ and $\quad a_{t}=1+$ $1.06 \ln \left(m_{S_{1}} / \mathrm{TeV}\right)$ [35]. Equation (4.13) then implies

$\operatorname{Re}\left(\tilde{Y}_{3}^{L} Y_{3}^{R *}\right) \gtrsim \frac{1-0.09 \ln \left(m_{S_{1}} / \mathrm{TeV}\right)}{1+1.06 \ln \left(m_{S_{1}} / \mathrm{TeV}\right)} \times 1.0 \times 10^{-3}\left(\frac{m_{S_{1}}}{\mathrm{TeV}}\right)^{2}$.

If seeking to obtain $\operatorname{Re}\left(\tilde{Y}_{3}^{L} Y_{3}^{R *}\right)$ in the simplest possible way, by generating a small contribution $Y_{3}^{R}=-\epsilon Y_{2}^{R}$, one must require $\epsilon \sim \mathcal{O}(1)$ to invalidate the strong $D^{0}$ constraint on the second-generation couplings. We plot in Fig. 1 the leptoquark-mass dependence of this minimally required $\epsilon$.

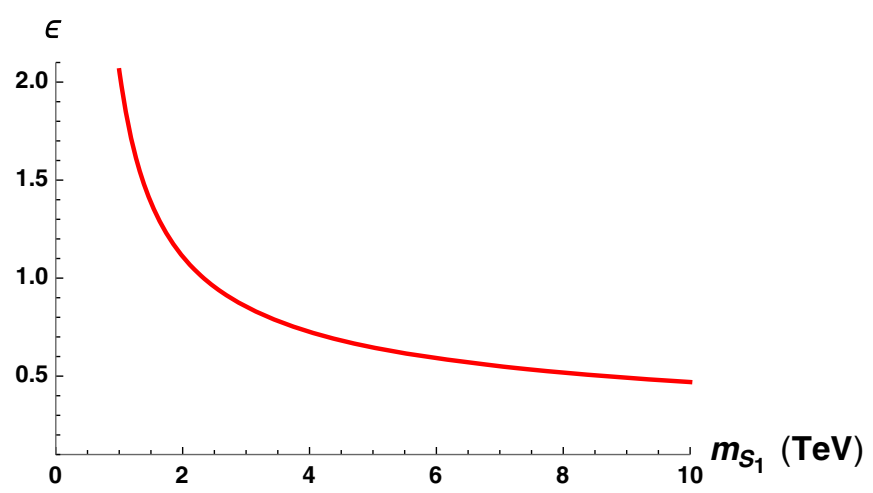

FIG. 1. The minimum value of $\epsilon=Y_{3}^{R} / Y_{2}^{R}$, as a function of mass $m_{S_{1}}$, required in Model 1 (down-origin case) to obtain the correct $\delta(g-2)_{\mu}$ and at the same time respect the 95\% C.L. $\operatorname{Br}\left(D^{0} \rightarrow \mu^{+} \mu^{-}\right)$bound. 
An equivalent calculation shows that in Model 2 one gets

$$
\begin{aligned}
\operatorname{Re}\left(\tilde{Y}_{3}^{R} Y_{3}^{L *}\right) \gtrsim & \frac{1-0.10 \ln \left(m_{R_{2}} / \mathrm{TeV}\right)}{1+0.61 \ln \left(m_{R_{2}} / \mathrm{TeV}\right)} \\
& \times 0.48 \times 10^{-3}\left(\frac{m_{R_{2}}}{\mathrm{TeV}}\right)^{2},
\end{aligned}
$$

which does not change the size of the required $\epsilon$ by much.

We point out finally that the measurement of $\operatorname{Br}\left(D^{0} \rightarrow\right.$ $\mu^{+} \mu^{-}$) at LHCb, Eq. (4.6), is already a few years old, and as such it is probably on track to be renewed with fresh data in the near future. If a new determination tightens the upper bound with respect to Eq. (4.6), then the minimally required top coupling is going to be even larger. ${ }^{1}$

\section{B. Up origin}

Flavor constraints provide a less clear-cut picture in the case of the up-origin Yukawa texture. As can be inferred from the form of Eqs. (3.3) and (3.7), in this case different flavor processes place bounds on the couplings of Model 1 and Model 2 separately.

In Model 1, the strongest bound can be derived from the measurement of the branching ratio $\operatorname{Br}\left(K^{+} \rightarrow \pi^{+} \nu \bar{\nu}\right)$ [70,71]. The observed value is $\operatorname{Br}\left(K^{+} \rightarrow \pi^{+} \nu \bar{\nu}\right)=$ $\left(1.73_{-1.05}^{+1.15}\right) \times 10^{-10}$, which was measured by the E949 Collaboration [72].

The effective Lagrangian relevant to this process can be written down as [71]

\footnotetext{
${ }^{1}$ The fact that we need third-generation couplings to explain $\delta(g-2)_{\mu}$ once the bound (4.13) is enforced raises the question of whether the scenarios investigated here can be made consistent with the recently observed flavor anomalies at $B A B A R$, Belle, and $\mathrm{LHCb}$, in particular the branching ratio measurements providing tantalizing hints of lepton-flavor nonuniversality [62-66]. Explanations of the observed excesses in $R_{D^{(*)}}=\operatorname{Br}\left(\bar{B} \rightarrow D^{(*)} \tau \nu_{\tau}\right) /$ $\operatorname{Br}\left(\bar{B} \rightarrow D^{(*)} l \nu_{l}\right)$ based on $S_{1}$ and $R_{2}$ seem to imply nonzero couplings between the leptoquark and the $\tau\left(\nu_{\tau}\right)$ (see, e.g., Refs. [29,35,52,58]), and thus lie outside of the main focus of this work. On the other hand, the deficits with respect to the SM observed in the $R_{K}$ and $R_{K^{*}}$ ratios can admit a viable explanation with couplings to the muon only. For example, the best-fit point to global analyses has been shown to imply, in Model $1, m_{S_{1}}=5.2 \mathrm{TeV}, \hat{Y}_{2}^{L}=0.15, \hat{Y}_{3}^{L} \approx-\sqrt{4 \pi}$, with $Y_{3}^{R} \approx Y_{2}^{R}$ very small or close to zero [52]. We find that this is consistent with $\delta(g-2)_{\mu}$ at $2 \sigma$, provided $\left|Y_{3}^{R}\right| \approx\left|Y_{2}^{R}\right| \gtrsim 0.005$. (Note, incidentally, that this can be regarded as a "topphilic" solution and thus a deeper investigation of its properties also somewhat exceeds the purpose of this paper.) The current LHC bounds from rare top decays are still unconstraining, implying $\left|\hat{Y}_{2}^{L} \hat{Y}_{3}^{L}\right| \lesssim$ $\mathcal{O}(1) \times\left(m_{S_{1}} / \mathrm{TeV}\right)^{2}[67,68]$. Conversely, the best-fit solution in Model 2 requires enforcing $\hat{Y}_{i=1,2,3}^{R} \approx 0$ to very high precision, in order to suppress the unwanted tree-level contributions to the Wilson coefficients $C_{9}$ and $C_{10}$, which would lead to $R_{K^{(*)}}>$ $R_{K^{(*)}}^{\mathrm{SM}}$ [69]. In the limit of zero right-handed couplings, the anomaly in $(g-2)_{\mu}$ cannot be explained.
}

$$
\mathcal{L}_{K \pi \nu \nu}=\sum_{\ell=e, \mu, \tau}\left(C_{V L L}^{K 1 \ell}+C_{\mathrm{SM}}^{K 1 \ell}\right)\left(\bar{d}_{L} \gamma^{\mu} s_{L}\right)\left(\bar{\nu}_{\ell L} \gamma_{\mu} \nu_{\ell L}\right)+\text { H.c. }
$$

where

$$
\begin{gathered}
C_{\mathrm{SM}}^{K 1 \ell}=-C_{F}\left[\lambda_{c} X_{N L}^{\ell}+\lambda_{t} X\left(x_{t}\right)\right] \\
C_{V L L}^{K 1 \ell}=\frac{1}{2 m_{S 1}^{2}} \hat{Y}_{1}^{L} \hat{Y}_{2}^{L *}=\frac{\lambda}{2 m_{S 1}^{2}}\left(1-\frac{\lambda^{2}}{2}\right)\left|\tilde{Y}_{2}^{L}\right|^{2} \delta_{\ell \mu}, \\
\lambda_{c(t)}=V_{c(t) s} V_{c(t) d}^{*}, X\left(x_{t}\right) \text { (with } x_{t}=m_{t}^{2} / m_{W}^{2} \text { ) is the top }
\end{gathered}
$$
quark loop contribution to the effective operator, $X_{N L}^{\ell}$ is the charm-lepton $\ell$ loop contribution, and

$$
C_{F}=\frac{4 G_{F}}{\sqrt{2}} \frac{\alpha}{2 \pi s_{W}^{2}} .
$$

Using the Wolfenstein parametrization one writes

$$
\begin{gathered}
\lambda_{c}=-\lambda\left(1-\frac{\lambda^{2}}{2}\right), \\
\lambda_{t}=-A^{2} \lambda^{5}(1-\rho+i \eta) .
\end{gathered}
$$

The charm contributions are numerically computed, e.g, in Refs. [73,74]. We use

$X_{N L}^{e}=X_{N L}^{\mu}=10.6 \times 10^{-4}, \quad X_{N L}^{\tau}=7.01 \times 10^{-4}$,

and the top loop function is

$$
X(x)=\frac{x}{8}\left(\frac{x+2}{x-1}+\frac{3(x-2)}{(1-x)^{2}} \ln x\right) .
$$

Finally, we write down the branching ratio:

$$
\begin{aligned}
\operatorname{Br} & \left.K^{+} \rightarrow \pi^{+} \nu \bar{\nu}\right) \\
= & \frac{\kappa_{+}}{\lambda^{10}}\left\{\operatorname{Im}\left[\lambda_{t}\right]^{2} X\left(x_{t}\right)^{2}+\frac{2}{3}\left(\operatorname{Re}\left[\lambda_{t}\right] X\left(x_{t}\right)+\lambda_{c} X_{N L}^{e}\right)^{2}\right. \\
& +\frac{1}{3}\left(\operatorname{Re}\left[\lambda_{t}\right] X\left(x_{t}\right)+\lambda_{c} X_{N L}^{\tau}\right)^{2} \\
& \left.+\frac{C_{V L L}^{K 1 \mu}}{3 C_{F}}\left[\frac{C_{V L L}^{K 1 \mu}}{C_{F}}-2\left(\operatorname{Re}\left[\lambda_{t}\right] X\left(x_{t}\right)+\lambda_{c} X_{N L}^{e}\right)\right]\right\},
\end{aligned}
$$

where $\kappa_{+}=5.27 \times 10^{-11}$ [75]. The first three terms in Eq. (4.25) yield the SM contribution, $\operatorname{Br}\left(K^{+} \rightarrow \pi^{+} \nu \bar{\nu}\right)_{\mathrm{SM}} \approx$ $9 \times 10^{-11}$. We get the $2 \sigma$ bound

$$
\left|\tilde{Y}_{2}^{L}\right|<5.26 \times 10^{-2}\left(\frac{m_{S 1}}{\mathrm{TeV}}\right) .
$$


TABLE III. The parameters used to obtain the experimental constraints from $\operatorname{Br}\left(K^{+} \rightarrow \pi^{+} \nu \bar{\nu}\right)$ and $\operatorname{Br}\left(K_{L} \rightarrow \mu^{+} \mu^{-}\right) . G_{F}, \alpha$ and $s_{W}^{2}$ are given by CODATA [60]. For the other constants, the PDG values are employed [61].

\begin{tabular}{lc}
\hline \hline$G_{F}$ & $1.17 \times 10^{-5} \mathrm{GeV}^{-2}$ \\
$\alpha$ & $1 / 137$ \\
$s_{W}^{2}$ & 0.231 \\
$A$ & 0.836 \\
$\rho$ & 0.135 \\
$E_{T}$ & 0.349 \\
$m_{t}$ & $173 \mathrm{GeV}$ \\
$m_{W}$ & $80.4 \mathrm{GeV}$ \\
\hline \hline$\tau_{K}$ & $5.12 \times 10^{-8} \mathrm{sec}$. \\
$m_{K}$ & $498 \mathrm{MeV}$ \\
$f_{K}$ & $156 \mathrm{MeV}$ \\
$m_{s}$ & $95 \mathrm{MeV}$ \\
\hline \hline
\end{tabular}

The parameters used to obtain the numerical constraints are shown in Table. III.

As we shall better see in Sec. V, the bound of Eq. (4.26) implies that large $R$-type couplings are required in Model 1 to be consistent with the BNL value for $\delta(g-2)_{\mu}$. Thus, the parameter space can be directly probed by the LHC.

In Model 2, the induced Yukawa coupling is restricted by the measurement of $\operatorname{Br}\left(K_{L} \rightarrow \mu^{+} \mu^{-}\right)$. This process is dominated by the long-distance contribution through $K_{L} \rightarrow \gamma \gamma$. The $90 \%$ C.L. upper bound on the short distance contribution is given in Ref. [76] as $\operatorname{Br}\left(K_{L} \rightarrow \mu^{+} \mu^{-}\right)_{\text {SD }}<$ $2.5 \times 10^{-9}$.

The process is generated by the following effective interaction [71]:

$$
\mathcal{L}_{K \mu \mu}=\left(C_{V L A}^{K 2}+C_{\mathrm{SM}}^{K 2}\right)\left(\bar{d}_{L} \gamma^{\mu} s_{L}\right)\left(\bar{\mu} \gamma_{\mu} \gamma_{5} \mu\right)+\text { H.c. },
$$

where

$$
\begin{gathered}
C_{\mathrm{SM}}^{K 2}=-\frac{C_{F}}{2}\left[\lambda_{c} Y_{N L}+\lambda_{t} Y\left(x_{t}\right)\right], \\
C_{V L A}^{K 2}=\frac{1}{4 m_{R_{2}}^{2}} \hat{Y}_{1}^{R} \hat{Y}_{2}^{R *}=\frac{\lambda}{4 m_{R_{2}}^{2}}\left(1-\frac{\lambda^{2}}{2}\right)\left|\tilde{Y}_{2}^{R}\right|^{2},
\end{gathered}
$$

with $Y_{N L}$ representing the contribution of loop diagrams involving the charm, and $Y\left(x_{t}\right)$ the contribution from top quark loops. The charm contribution is $Y_{N L}=3.50 \times 10^{-4}$ [73], and the top loop function is

$$
Y(x)=\frac{x}{8}\left(\frac{x-4}{x-1}+\frac{3 x}{(1-x)^{2}} \ln x\right)
$$

Neglecting the tiny $C P$-violating contribution, we write down the short-distance branching ratio:

$$
\begin{aligned}
\operatorname{Br}\left(K_{L} \rightarrow \mu^{+} \mu^{-}\right)_{\mathrm{SD}}= & \tau_{K} \frac{f_{K}^{2} m_{K}^{3}}{4 \pi} \frac{m_{\mu}^{2}}{m_{K}^{2}} \sqrt{1-\frac{4 m_{\mu}^{2}}{m_{K}^{2}}} \\
& \times \operatorname{Re}\left(C_{V L A}^{K 2}+2 C_{\mathrm{SM}}^{K 2}\right)^{2},
\end{aligned}
$$

where lifetime $\tau_{K}$ and other constants relevant for the calculation are given in Table III. The SM contribution is $\operatorname{Br}\left(K_{L} \rightarrow \mu^{+} \mu^{-}\right)_{\mathrm{SM}}=1.08 \times 10^{-9}$. As a result, we obtain the following constraint:

$$
\left|\tilde{Y}_{2}^{R}\right|<1.9 \times 10^{-2}\left(\frac{m_{R_{2}}}{\mathrm{TeV}}\right)
$$

We conclude this section with a note on EW precision bounds. Precision tests of the EW theory are not very constraining for charmphilic leptoquark scenarios. The presence of leptoquarks can modify the coupling $Z \rightarrow f \bar{f}$ and induce effects that can be picked up in data from the $Z$ line shape and asymmetry observables. The analytical form of the corresponding loop contribution can be found, for example, in [77]. Since, in the charmphilic assumption, the leptoquark couples only to the second generation of fermions, a loop correction to the effective $Z \rightarrow f \bar{f}$ coupling scales proportionally to the $Z$ boson threshold, $\sim\left|Y_{2}^{R, L}\right|{ }^{2} m_{Z}^{2} / m_{S_{1}, R_{2}}^{2}$. Therefore, it does not introduce additional constraints on the parameter space allowed by $(g-2)_{\mu}$ and other flavor observables.

\section{LHC CONSTRAINTS ON THE PARAMETER SPACE}

The parameter space surviving flavor exclusion bounds in charmphilic leptoquark scenarios for $(g-2)_{\mu}$ falls squarely inside the reach of LHC searches. At small-tomoderate Yukawa couplings, $Y \sim \mathcal{O}(0.1)$, leptoquarks are predominantly pair-produced with a cross section directly proportional to the QCD strong coupling. Therefore, in this case the collider exclusion bounds depend solely on the mass of the leptoquark $m_{\mathrm{LQ}}$ and read $m_{\mathrm{LQ}} \gtrsim 1.5 \mathrm{TeV}$ [78-80].

For larger Yukawa couplings, $Y \sim \mathcal{O}(1)$, dilepton production with a $t$-channel leptoquark exchange, as well as single leptoquark production, can be directly probed over vast mass ranges, and provide complementary constraints $[81,82]$. Very recently it was also pointed out [83] that, for leptoquarks coupling to the first quark generation in particular, monolepton searches can provide bounds equivalent to those from dilepton. In the large mass regime, where QCD direct production saturates, dilepton signatures are particularly effective in constraining the second-generation couplings. Generation-dependent constraints were recently provided in Refs. $[51,52,84]$ by recasting the results of two $13 \mathrm{TeV}$ LHC searches for high-mass resonances in dilepton final state by the ATLAS 


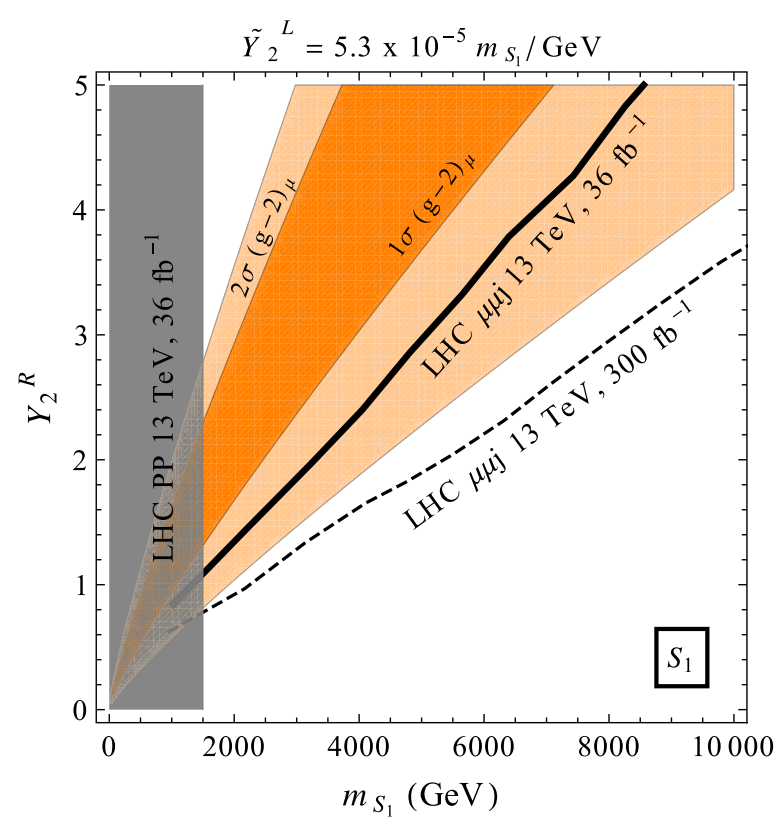

(a)

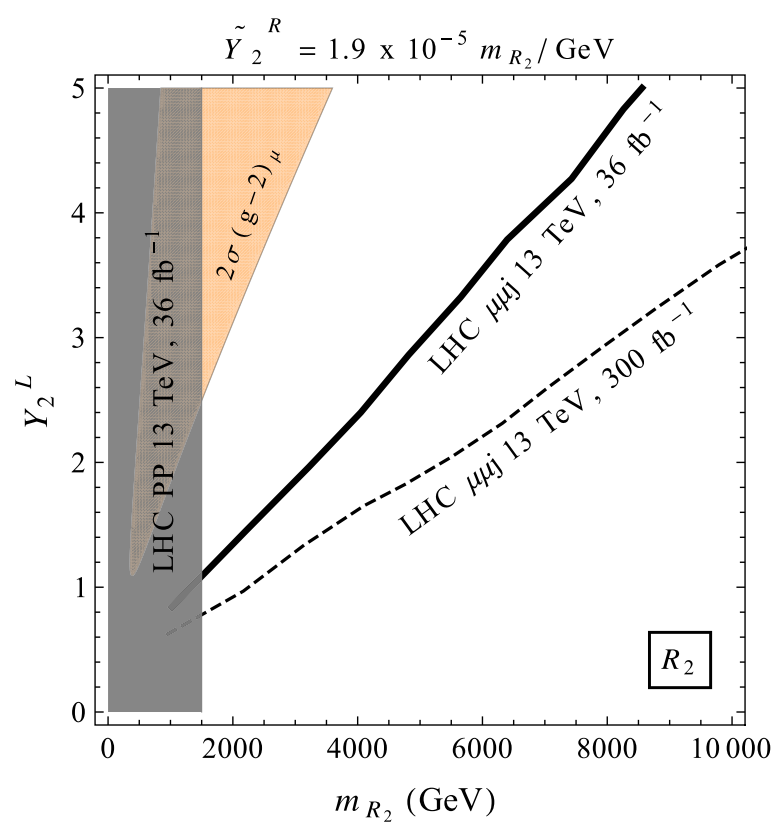

(b)

FIG. 2. (a) Parameter space of the leptoquark model $S_{1}$ in the plane $\left(m_{S_{1}}, Y_{2}^{R}\right)$ allowed by the measurement of $\delta(g-2)_{\mu}$ and the experimental bounds from $K^{+} \rightarrow \pi^{+} \nu \bar{\nu}$. The $1 \sigma(2 \sigma)$ region is shown in orange (light orange). Solid black line indicates the $95 \%$ C.L. upper limit based on our recast of the CMS dilepton search at $36 \mathrm{fb}^{-1}$ [42], while the dashed black line indicates the projected sensitivity for $300 \mathrm{fb}^{-1}$. Gray region represents the $95 \%$ C.L. exclusion bound due to the leptoquark pair-production [78-80]. (b) Same for the parameter space of the leptoquark model $R_{2}$ in the plane $\left(m_{R_{2}}, Y_{2}^{L}\right)$ allowed by the measurement of $\delta(g-2)_{\mu}$ and the experimental bounds from the short-distance contribution to $K_{L} \rightarrow \mu^{+} \mu^{-}$.

Collaboration [41] and the CMS Collaboration [42] for a large set of leptoquark models.

We perform an analysis along similar lines in this study, and apply it to the parameter space of the $S_{1}$ and $R_{2}$ models, allowed after incorporating the bounds from the measurement of $(g-2)_{\mu}$ and other flavor observables. Recall, in particular, that while we have shown that the down-origin case for $\delta(g-2)_{\mu}$ is excluded by the measurement of $\operatorname{Br}\left(D^{0} \rightarrow \mu^{+} \mu^{-}\right)$, when it comes to the up-origin assumption Eqs. (4.26) and (4.32) only bound one of the couplings entering the product $\operatorname{Re}\left(g_{L} g_{R}^{*}\right)$ in Eq. (2.7). The remaining part corresponds to relatively large values of the Yukawa couplings $Y_{2}^{R}, Y_{2}^{L}$, and hence it is subject to constraints from the LHC dimuon searches.

To perform the analysis, each leptoquark model was generated with FeynRules [85] and the corresponding UFO files were passed to MadGraph5_aMC@NLO [86]. The results were cross-checked with the code used in [20], based on SPheno [87,88], PYTHIA [89], and DELPHES 3 [90]. We generated two process, $p p \rightarrow \mu^{+} \mu^{-}$and $p p \rightarrow$ $\mu^{+} \mu^{-} j$, particularly the $g^{(-)} c \rightarrow \mu^{+} \mu^{-} \stackrel{(-)}{c}$ contribution (without the $c$-tag), which can produce a signal in dimuon searches, because jets in the generated events are not vetoed. Note that the inclusion of $p p \rightarrow \mu^{+} \mu^{-} j$ is particularly important, as in this case the invariant mass distribution acquires a long high- $p_{T}$ tail due to the gluon PDF.
This effect cannot be observed in the pure $p p \rightarrow \mu^{+} \mu^{-}$ production.

Six kinematical bins based on the invariant dimuon mass, $m_{\mu \mu}$, were constructed, closely following the CMS search for high-mass resonances in dilepton final states [42]. For each point in the parameter space we calculate the likelihood function, using a Poisson distribution smeared with the experimental background determination uncertainty provided in Ref. [42], and statistically combining the six exclusive kinematical bins of the invariant dimuon mass by multiplying the individual likelihood functions. The 95\% C.L. exclusion limit was derived using the $\Delta \chi^{2}$ statistics. The dominant bins affecting the obtained bound were the three highest by invariant mass, $m_{\mu \mu}>900 \mathrm{GeV}$.

We show in Fig. 2(a) the 95\% C.L. upper bound from the LHC in solid black. ${ }^{2}$ Gray region represents the present 95\% C.L. exclusion due to leptoquark pair-production [78-80]. In dark orange we show the $1 \sigma$ region for $\delta(g-2)_{\mu}$ in Model 1 after the strongest flavor constraint, Eq. (4.26), has been taken into account. We show in light orange the corresponding $2 \sigma$ region. The dashed black line

\footnotetext{
${ }^{2}$ Our $36 \mathrm{fb}^{-1}$ bound is in good agreement with the recent estimate of Ref. [52] after we include the process $p p \rightarrow \mu^{+} \mu^{-} j$ in our simulation. If one only includes $p p(c \bar{c}) \rightarrow \mu^{+} \mu^{-}$the bound weakens, and it agrees with the recent computation of Ref. [51].
} 


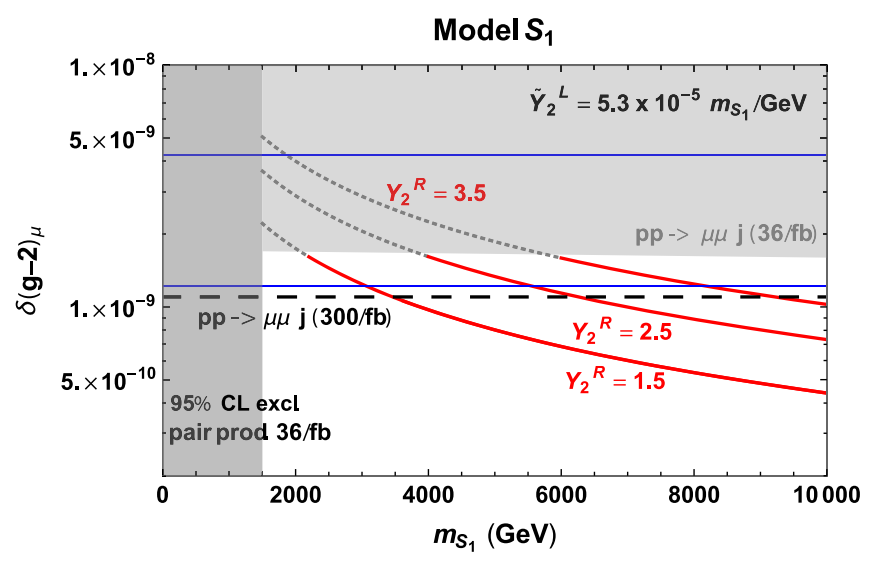

FIG. 3. The value of $\delta(g-2)_{\mu}$ after including the bounds from $\operatorname{Br}\left(K^{+} \rightarrow \pi^{+} \nu \bar{\nu}\right)$, as a function of leptoquark mass $m_{S_{1}}$, confronted with existing and projected LHC bounds. The solid blue lines delimit the $2 \sigma$ region of the BNL experiment. The dark gray region shows the lower mass bound from Refs. [78-80]. In light gray, we present our recasting of the dilepton bound from Ref. [42], applied to $g\left({ }_{c}^{(-)} \rightarrow \mu^{+} \mu^{-(-)}\right.$c processes. Our projection for $300 \mathrm{fb}^{-1}$ is shown as a black dashed line.

indicates our projected LHC sensitivity with $300 \mathrm{fb}^{-1}$, which entirely excludes the $2 \sigma$ region. Note, moreover, that the currently running NA62 experiment is expected to improve the $\operatorname{Br}\left(K^{+} \rightarrow \pi^{+} \nu \bar{\nu}\right)$ measurement down to a precision of about $10 \%$ [91] and thus further tighten the bounds on this scenario.

In Fig. 2(b) we compare our 95\% C.L. upper bound (black solid) with the allowed $2 \sigma$ parameter space (light orange) in Model 2, after the constraint from Eq. (4.32) has been taken into account. The model is already excluded by the LHC. In order to explain the $(g-2)_{\mu}$ anomaly and at the same time avoid the strong tension with this bound one would need an additional coupling to the top quark of the size of the coupling to the charm. Accidentally, the minimally required $\operatorname{Re}\left(\tilde{Y}_{3}^{R} Y_{3}^{L *}\right)$ in this case coincides with the amount required to evade the $D^{0} \rightarrow \mu^{+} \mu^{-}$bound in the down-origin scenario, Eq. (4.16).

Finally we summarize the overall collider situation in Fig. 3, which shows a plot of the deviation from the SM, $\delta(g-2)_{\mu}$, as a function of leptoquark mass in Model 1, confronted with our calculated LHC bounds and projections. One can see that the reach of the LHC searches pushes down along the $y$-axis as the luminosity increases. Thus, the LHC will continue to provide, with its HighLuminosity run, the most effective tool to probe these scenarios for a wide range of possible outcomes at Fermilab.

\section{SUMMARY AND CONCLUSIONS}

We have considered in this work charmphilic contributions to the muon anomalous magnetic moment with a single leptoquark. As the only way to increase the value of $(g-2)_{\mu}$, and at the same time keep the leptoquark mass beyond direct-production bounds at colliders, is in this case to couple to the quark with a log-enhanced interaction, only two models are relevant for this observable: the scalar leptoquarks $S_{1}$ and $R_{2}$. We have confronted these two models with relevant constraints from flavor measurements and numerically recast the LHC searches that can provide applicable limits.

In order to systematically address the flavor bounds, we have subdivided the available parameter space of these models into two regions: "up-origin," if the charmphilic ansatz applies to the leptoquark coupling to the muon and to the up-type component of the second generation quark doublet, and the "down-origin," if it applies to the coupling with the muon and the down-type component quark.

We find that, under the down-origin assumption, the parameter space consistent with the measured $(g-2)_{\mu}$ anomaly at BNL is in both models entirely excluded by the measurement of $\operatorname{Br}\left(D^{0} \rightarrow \mu^{+} \mu^{-}\right)$, unless one introduces a coupling to the top quark of the same order of magnitude as the charm's.

Conversely, under the up-origin assumption some of the parameter space of the model with $R_{2}$ survives the most constraining flavor bound, from the short-distance contribution to $\operatorname{Br}\left(K_{L} \rightarrow \mu^{+} \mu^{-}\right)$, but is by now entirely excluded by searches with two muons in the final state at the LHC. The bound can be evaded by assuming the existence of couplings of both the chiral states of the muon to the top quark, of approximately the same size as the couplings to the charm.

Finally, the leptoquark $S_{1}$ is the least constrained under the up-origin assumption. Some of the parameter space survives the most constraining flavor bound, from $\operatorname{Br}\left(K^{+} \rightarrow \pi^{+} \nu \bar{\nu}\right)$, and also some of the BNL $2 \sigma$ region remains in play after the LHC dimuon searches are taken into account. However, once $300 \mathrm{fb}^{-1}$ of luminosity are accumulated, the remaining parameter space will be probed in its entirety.

\section{ACKNOWLEDGMENTS}

We would like to thank K. Sakurai for his comments on the manuscript. Y. Y. would like to thank M. Tanaka and K. Hamaguchi for directing his interest to leptoquarks and the muon $\mathrm{g}-2$. K. K. is supported in part by the National Science Centre (Poland) under the research Grant No. 2017/26/E/ST2/00470. E. M. S. and Y. Y. are supported in part by the National Science Centre (Poland) under the research Grant No. 2017/26/D/ ST2/00490. The use of the CIS computer cluster at the National Centre for Nuclear Research in Warsaw is gratefully acknowledged. 
[1] J. Grange et al. (Muon g-2 Collaboration), Muon (g-2) technical design report, arXiv:1501.06858.

[2] T. Mibe (J-PARC g-2 Collaboration), New g-2 experiment at J-PARC, Chin. Phys. C 34, 745 (2010).

[3] G. W. Bennett et al. (Muon g-2 Collaboration), Final report of the Muon E821 anomalous magnetic moment measurement at BNL, Phys. Rev. D 73, 072003 (2006).

[4] M. Davier, Update of the hadronic vacuum polarisation contribution to the muon g-2, Nucl. Part. Phys. Proc. 287288, 70 (2017).

[5] F. Jegerlehner, Muon g-2 theory: The hadronic part, EPJ Web Conf. 166, 00022 (2018).

[6] T. Moroi, The Muon anomalous magnetic dipole moment in the minimal supersymmetric standard model, Phys. Rev. D 53, 6565 (1996); Erratum, Phys. Rev. D56, 4424(E) (1997).

[7] G.-C. Cho, K. Hagiwara, and M. Hayakawa, Muon g-2 and precision electroweak physics in the MSSM, Phys. Lett. B 478, 231 (2000).

[8] S. P. Martin and J. D. Wells, Muon anomalous magnetic dipole moment in supersymmetric theories, Phys. Rev. D 64, 035003 (2001).

[9] M. Aaboud et al. (ATLAS Collaboration), Search for electroweak production of supersymmetric particles in final states with two or three leptons at $\sqrt{s}=13 \mathrm{TeV}$ with the ATLAS detector, Eur. Phys. J. C 78, 995 (2018).

[10] M. Aaboud et al. (ATLAS Collaboration), Search for electroweak production of supersymmetric states in scenarios with compressed mass spectra at $\sqrt{s}=13 \mathrm{TeV}$ with the ATLAS detector, Phys. Rev. D 97, 052010 (2018).

[11] A. M. Sirunyan et al. (CMS Collaboration), Search for electroweak production of charginos and neutralinos in multilepton final states in proton-proton collisions at $\sqrt{s}=13 \mathrm{TeV}$, J. High Energy Phys. 03 (2018) 166.

[12] A. M. Sirunyan et al. (CMS Collaboration), Search for new physics in events with two soft oppositely charged leptons and missing transverse momentum in proton-proton collisions at $\sqrt{s}=13 \mathrm{TeV}$, Phys. Lett. B 782, 440 (2018).

[13] M. Endo, K. Hamaguchi, S. Iwamoto, and T. Yoshinaga, Muon g-2 vs LHC in supersymmetric models, J. High Energy Phys. 01 (2014) 123.

[14] S. Akula and P. Nath, Gluino-driven radiative breaking, Higgs boson mass, muon g-2, and the Higgs diphoton decay in supergravity unification, Phys. Rev. D 87, 115022 (2013).

[15] A. Fowlie, K. Kowalska, L. Roszkowski, E. M. Sessolo, and Y.-L.S. Tsai, Dark matter and collider signatures of the MSSM, Phys. Rev. D 88, 055012 (2013).

[16] M. Endo, K. Hamaguchi, T. Kitahara, and T. Yoshinaga, Probing Bino contribution to Muon $g-2$, J. High Energy Phys. 11 (2013) 013.

[17] M. Chakraborti, U. Chattopadhyay, A. Choudhury, A. Datta, and S. Poddar, The electroweak sector of the pMSSM in the light of LHC-8 TeV and other data, J. High Energy Phys. 07 (2014) 019.

[18] K. Kowalska, L. Roszkowski, E. M. Sessolo, and A. J. Williams, GUT-inspired SUSY and the Muon $g-2$ anomaly: Prospects for LHC $14 \mathrm{TeV}$, J. High Energy Phys. 06 (2015) 020.

[19] B. P. Padley, K. Sinha, and K. Wang, Natural supersymmetry, Muon $g-2$, and the last crevices for the top squark, Phys. Rev. D 92, 055025 (2015).
[20] K. Kowalska and E. M. Sessolo, Expectations for the Muon $g-2$ in simplified models with dark matter, J. High Energy Phys. 09 (2017) 112.

[21] R. Dermisek and A. Raval, Explanation of the Muon $g-2$ anomaly with vectorlike leptons and its implications for Higgs decays, Phys. Rev. D 88, 013017 (2013).

[22] A. Freitas, J. Lykken, S. Kell, and S. Westhoff, Testing the Muon $g-2$ anomaly at the LHC, J. High Energy Phys. 05 (2014) 145; Erratum, J. High Energy Phys.09 (2014) 155(E).

[23] ATLAS Collaboration, Search for new physics in events with three charged leptons with the ATLAS detector, Technical Report No. ATLAS-CONF-2013-070, CERN, Geneva, 2013, http://cds.cern.ch/record/1562898.

[24] G. Aad et al. (ATLAS Collaboration), Search for new phenomena in events with three or more charged leptons in $p p$ collisions at $\sqrt{s}=8 \mathrm{TeV}$ with the ATLAS detector, J. High Energy Phys. 08 (2015) 138.

[25] A. Djouadi, T. Kohler, M. Spira, and J. Tutas, (e b), (e t) type leptoquarks at e p colliders, Z. Phys. C 46, 679 (1990).

[26] D. Chakraverty, D. Choudhury, and A. Datta, A nonsupersymmetric resolution of the anomalous muon magnetic moment, Phys. Lett. B 506, 103 (2001).

[27] K.-m. Cheung, Muon anomalous magnetic moment and leptoquark solutions, Phys. Rev. D 64, 033001 (2001).

[28] F. S. Queiroz and W. Shepherd, New physics contributions to the Muon anomalous magnetic moment: A numerical code, Phys. Rev. D 89, 095024 (2014).

[29] Y. Sakaki, M. Tanaka, A. Tayduganov, and R. Watanabe, Testing leptoquark models in $\bar{B} \rightarrow D^{(*)} \tau \bar{\nu}$, Phys. Rev. D 88, 094012 (2013).

[30] G. Hiller and M. Schmaltz, $R_{K}$ and future $b \rightarrow s \ell \ell$ physics beyond the standard model opportunities, Phys. Rev. D 90, 054014 (2014).

[31] B. Gripaios, M. Nardecchia, and S. A. Renner, Composite leptoquarks and anomalies in $B$-meson decays, J. High Energy Phys. 05 (2015) 006.

[32] I. de Medeiros Varzielas and G. Hiller, Clues for flavor from rare lepton and quark decays, J. High Energy Phys. 06 (2015) 072.

[33] L. Calibbi, A. Crivellin, and T. Ota, Effective Field Theory Approach to $b \rightarrow s l l^{\prime}, B \rightarrow K^{(*)} \nu \bar{\nu}$ and $B \rightarrow D^{(*)} \tau \nu$ with Third Generation Couplings, Phys. Rev. Lett. 115, 181801 (2015).

[34] M. Freytsis, Z. Ligeti, and J. T. Ruderman, Flavor models for $\bar{B} \rightarrow D^{(*)} \tau \bar{\nu}$, Phys. Rev. D 92, 054018 (2015).

[35] M. Bauer and M. Neubert, Minimal Leptoquark Explanation for the $\mathbf{R}_{D^{(*)}}, \mathbf{R}_{K}$, and $(g-2)_{g}$ Anomalies, Phys. Rev. Lett. 116, 141802 (2016).

[36] S. Fajfer and N. Košnik, Vector leptoquark resolution of $R_{K}$ and $R_{D^{(*)}}$ puzzles, Phys. Lett. B 755, 270 (2016).

[37] R. Barbieri, G. Isidori, A. Pattori, and F. Senia, Anomalies in $B$-decays and $U(2)$ flavour symmetry, Eur. Phys. J. C 76, 67 (2016).

[38] E. Coluccio Leskow, G. D'Ambrosio, A. Crivellin, and D. Müller, $(g-2) \mu$, lepton flavor violation, and $Z$ decays with leptoquarks: Correlations and future prospects, Phys. Rev. D 95, 055018 (2017).

[39] S. Bar-Shalom, J. Cohen, A. Soni, and J. Wudka, Phenomenology of TeV-scale scalar Leptoquarks in the EFT, arXiv:1812.03178. 
[40] L. Calibbi, R. Ziegler, and J. Zupan, Minimal models for dark matter and the Muon $g-2$ anomaly, J. High Energy Phys. 07 (2018) 046.

[41] M. Aaboud et al. (ATLAS Collaboration), Search for new high-mass phenomena in the dilepton final state using $36 \mathrm{fb}^{1}$ of proton-proton collision data at $\sqrt{s}=13 \mathrm{TeV}$ with the ATLAS detector, J. High Energy Phys. 10 (2017) 182.

[42] A. M. Sirunyan et al. (CMS Collaboration), Search for highmass resonances in dilepton final states in proton-proton collisions at $\sqrt{s}=13 \mathrm{TeV}$, J. High Energy Phys. 06 (2018) 120.

[43] E. Eichten, I. Hinchliffe, K. D. Lane, and C. Quigg, Signatures for technicolor, Phys. Rev. D 34, 1547 (1986).

[44] K. D. Lane and M. V. Ramana, Walking technicolor signatures at hadron colliders, Phys. Rev. D 44, 2678 (1991).

[45] N. Assad, B. Fornal, and B. Grinstein, Baryon number and lepton universality violation in leptoquark and diquark models, Phys. Lett. B 777, 324 (2018).

[46] L. Di Luzio, A. Greljo, and M. Nardecchia, Gauge leptoquark as the origin of B-physics anomalies, Phys. Rev. D 96, 115011 (2017).

[47] L. Calibbi, A. Crivellin, and T. Li, A model of vector leptoquarks in view of the $B$-physics anomalies, Phys. Rev. D 98, 115002 (2018).

[48] M. Bordone, C. Cornella, J. Fuentes-Martin, and G. Isidori, A three-site gauge model for flavor hierarchies and flavor anomalies, Phys. Lett. B 779, 317 (2018).

[49] B. Fornal, S. A. Gadam, and B. Grinstein, Left-right SU(4) vector leptoquark model for flavor anomalies, arXiv:1812 .01603 .

[50] A. M. Sirunyan et al. (CMS Collaboration), Constraints on models of scalar and vector leptoquarks decaying to a quark and a neutrino at $\sqrt{s}=13 \mathrm{TeV}$, Phys. Rev. D 98, 032005 (2018).

[51] M. Schmaltz and Y.-M. Zhong, The leptoquark Hunter's guide: Large coupling, J. High Energy Phys. 01 (2019) 132.

[52] A. Angelescu, D. Beirevi, D. A. Faroughy, and O. Sumensari, Closing the window on single leptoquark solutions to the $B$-physics anomalies, J. High Energy Phys. 10 (2018) 183.

[53] E. Keith and E. Ma, S, T, and Leptoquarks at HERA, Phys. Rev. Lett. 79, 4318 (1997).

[54] M. Baak, J. Cth, J. Haller, A. Hoecker, R. Kogler, K. Mnig, M. Schott, and J. Stelzer (Gfitter Group Collaboration), The global electroweak fit at NNLO and prospects for the LHC and ILC, Eur. Phys. J. C 74, 3046 (2014).

[55] S. de Boer and G. Hiller, Flavor and new physics opportunities with rare charm decays into leptons, Phys. Rev. D 93, 074001 (2016).

[56] R. Aaij et al. (LHCb Collaboration), Search for the rare decay $D^{0} \rightarrow \mu^{+} \mu^{-}$, Phys. Lett. B 725, 15 (2013).

[57] S. Fajfer and N. Košnik, Prospects of discovering new physics in rare charm decays, Eur. Phys. J. C 75, 567 (2015).

[58] Y. Cai, J. Gargalionis, M. A. Schmidt, and R. R. Volkas, Reconsidering the one Leptoquark solution: Flavor anomalies and neutrino mass, J. High Energy Phys. 10 (2017) 047.

[59] A. Paul, I. I. Bigi, and S. Recksiegel, $D^{0} \rightarrow \gamma \gamma$ and $D^{0} \rightarrow$ $\mu^{+} \mu^{-}$rates on an unlikely impact of the littlest Higgs model with T-parity, Phys. Rev. D 82, 094006 (2010); Erratum, Phys. Rev. D83, 019901(E) (2011).

[60] P. J. Mohr, D. B. Newell, and B. N. Taylor, CODATA recommended values of the fundamental physical constants: 2014, Rev. Mod. Phys. 88, 035009 (2016).

[61] M. Tanabashi et al. (Particle Data Group), Review of particle physics, Phys. Rev. D 98, 030001 (2018).

[62] J. P. Lees et al. (BABAR Collaboration), Measurement of an excess of $\bar{B} \rightarrow D^{(*)} \tau^{-} \bar{\nu}_{\tau}$ decays and implications for charged Higgs bosons, Phys. Rev. D 88, 072012 (2013).

[63] R. Aaij et al. (LHCb Collaboration), Measurement of the Ratio of Branching Fractions $\mathcal{B}\left(\bar{B}^{0} \rightarrow D^{*+} \tau^{-} \bar{\nu}_{\tau}\right) /$ $\mathcal{B}\left(\bar{B}^{0} \rightarrow D^{*+} \mu^{-} \bar{\nu}_{\mu}\right)$, Phys. Rev. Lett. 115, 111803 (2015); Erratum, Phys. Rev. Lett.115, 159901(E) (2015).

[64] S. Hirose et al. (Belle Collaboration), Measurement of the $\tau$ Lepton Polarization and $R\left(D^{*}\right)$ in the Decay $\bar{B} \rightarrow D^{*} \tau^{-} \bar{\nu}_{\tau}$, Phys. Rev. Lett. 118, 211801 (2017).

[65] R. Aaij et al. (LHCb Collaboration), Test of Lepton Universality Using $\mathrm{B}^{+} \rightarrow \mathrm{K}^{+} \ell^{+} \ell^{-}$Decays, Phys. Rev. Lett. 113, 151601 (2014).

[66] R. Aaij et al. (LHCb Collaboration), Test of lepton universality with $B^{0} \rightarrow K^{* 0} \ell^{+} \ell^{-}$decays, J. High Energy Phys. 08 (2017) 055.

[67] G. Durieux, F. Maltoni, and C. Zhang, Global approach to top-quark flavor-changing interactions, Phys. Rev. D 91, 074017 (2015).

[68] M. Chala, J. Santiago, and M. Spannowsky, Constraining four-fermion operators using rare top decays, arXiv:1809 .09624 .

[69] D. Bečirević and O. Sumensari, A leptoquark model to accommodate $R_{K}^{\exp }<R_{K}^{\mathrm{SM}}$ and $R_{K^{*}}^{\exp }<R_{K^{*}}^{\mathrm{SM}}$, J. High Energy Phys. 08 (2017) 104.

[70] A. J. Buras, J. Girrbach-Noe, C. Niehoff, and D. M. Straub, $B \rightarrow K^{(*)} \nu \bar{\nu}$ decays in the Standard Model and beyond, J. High Energy Phys. 02 (2015) 184.

[71] G. Kumar, Constraints on a scalar leptoquark from the kaon sector, Phys. Rev. D 94, 014022 (2016).

[72] A. V. Artamonov et al. (E949 Collaboration), New Measurement of the $K^{+} \rightarrow \pi^{+} \nu \bar{\nu}$ Branching Ratio, Phys. Rev. Lett. 101, 191802 (2008).

[73] G. Buchalla, A. J. Buras, and M. E. Lautenbacher, Weak decays beyond leading logarithms, Rev. Mod. Phys. 68, 1125 (1996).

[74] J. Brod and M. Gorbahn, Electroweak corrections to the charm quark contribution to $K^{+} \rightarrow \pi^{+} \nu \bar{\nu}$, Phys. Rev. D 78, 034006 (2008).

[75] W. Altmannshofer, A. J. Buras, D. M. Straub, and M. Wick, New strategies for new physics search in $B \rightarrow K^{*} \nu \bar{\nu}$, $B \rightarrow K \nu \bar{\nu}$ and $B \rightarrow X_{s} \nu \bar{\nu}$ decays, J. High Energy Phys. 04 (2009) 022.

[76] G. Isidori and R. Unterdorfer, On the short distance constraints from $K_{L, S} \rightarrow \mu^{+} \mu^{-}$, J. High Energy Phys. 01 (2004) 009.

[77] S. Bansal, R. M. Capdevilla, and C. Kolda, On the minimal flavor violating leptoquark explanation of the $R_{D^{(*)}}$ anomaly, arXiv:1810.11588.

[78] M. Aaboud et al. (ATLAS Collaboration), Search for scalar leptoquarks in pp collisions at $\sqrt{s}=13 \mathrm{TeV}$ with the ATLAS experiment, New J. Phys. 18, 093016 (2016). 
[79] B. Diaz, M. Schmaltz, and Y.-M. Zhong, The leptoquark Hunters guide: Pair production, J. High Energy Phys. 10 (2017) 097.

[80] A. M. Sirunyan et al. (CMS Collaboration), Search for pair production of second-generation leptoquarks at $\sqrt{s}=13 \mathrm{TeV}$, arXiv:1808.05082.

[81] D. A. Faroughy, A. Greljo, and J. F. Kamenik, Confronting lepton flavor universality violation in B decays with high- $p_{T}$ tau lepton searches at LHC, Phys. Lett. B 764, 126 (2017).

[82] A. Greljo and D. Marzocca, High- $p_{T}$ dilepton tails and flavor physics, Eur. Phys. J. C 77, 548 (2017).

[83] S. Bansal, R. M. Capdevilla, A. Delgado, C. Kolda, A. Martin, and N. Raj, Hunting leptoquarks in monolepton searches, Phys. Rev. D 98, 015037 (2018).

[84] N. Raj, Anticipating nonresonant new physics in dilepton angular spectra at the LHC, Phys. Rev. D 95, 015011 (2017).

[85] A. Alloul, N. D. Christensen, C. Degrande, C. Duhr, and B. Fuks, FeynRules 2.0: A complete toolbox for tree-level phenomenology, Comput. Phys. Commun. 185, 2250 (2014).

[86] J. Alwall, R. Frederix, S. Frixione, V. Hirschi, F. Maltoni, O. Mattelaer, H. S. Shao, T. Stelzer, P. Torrielli, and M. Zaro, The automated computation of tree-level and next-to-leading order differential cross sections, and their matching to parton shower simulations, J. High Energy Phys. 07 (2014) 079.

[87] W. Porod, SPheno: A program for calculating supersymmetric spectra, SUSY particle decays and SUSY particle production at $e^{+} e^{-}$colliders, Comput. Phys. Commun. 153, 275 (2003).

[88] W. Porod and F. Staub, SPheno 3.1: Extensions including flavour, CP-phases and models beyond the MSSM, Comput. Phys. Commun. 183, 2458 (2012).

[89] T. Sjostrand, S. Mrenna, and P.Z. Skands, A brief introduction to PYTHIA 8.1, Comput. Phys. Commun. 178, 852 (2008).

[90] J. de Favereau, C. Delaere, P. Demin, A. Giammanco, V. Lematre, A. Mertens, and M. Selvaggi (DELPHES 3 Collaboration), DELPHES 3: A modular framework for fast simulation of a generic collider experiment, J. High Energy Phys. 02 (2014) 057.

[91] G. Anelli et al., Proposal to measure the rare decay $K^{+} \rightarrow \pi^{+} \nu \bar{\nu}$ at the CERN SPS, Reports No. CERNSPSC-2005-013, CERN-SPSC-P-326. 